\title{
Confusion in a Patient with Carcinoid Syndrome - A Case Report
}

\author{
Dr Vikram G Aarella - Kings Mill Hospital, Sutton in Ashfield; Dr Hannah Dix - Nevill Hall Hospital, Abergavenny; \\ Dr Stuart Lee - Wrexham Maelor Hospital, Wrexham
}

\begin{abstract}
Introduction
Confused patients are encountered frequently in the acute medical setting so it is important to have a good understanding of the causes of confusion and a good screening tool for identifying them. Common causes of confusion include infection, pain, cerebral pathology, metabolic disturbances, vitamin deficiencies, drug reactions and withdrawals or simply changes in surroundings. In the following case study we look at a rare cause of confusion that is associated with vitamin deficiency.
\end{abstract}

\section{Case Report}

A 64 year old lady was admitted with a two day history of feeling generally unwell. She complained of increased tiredness, sweating and flushing, accompanied by generalised abdominal pain. Her relatives also reported new onset confusion over these two days.

On examination the patient was despondent, confused, flushed and clammy. Her mood was particularly low, stating that she would be 'better off dead'. She was apyrexial, tachypnoeic, tachycardic and was displaying fluctuating blood pressures between hypotensive with an impalpable radial pulse and overtly hypertensive. She had a GCS of fourteen but was not orientated to time place or person. Physical examination revealed marked tender hepatomegaly and generalised abdominal tenderness. There was also marked peripheral oedema.

Her bloods showed raised inflammatory markers (CRP $110.3 \mathrm{mg} / \mathrm{L}$, WCC $18.2 \times 109 / \mathrm{L})$ hypoalbuminemia $(17 \mathrm{~g} / \mathrm{L})$, bilirubinemia $(55 \mathrm{umol} / \mathrm{L})$ and a raised alkaline phosphatase (917 IU/L).

She had recently been diagnosed with carcinoid, primary tumour situated in the terminal ileum and liver metastasise and had been deemed unfit for intervention by the specialist carcinoid team in Liverpool. She also suffered from chronic pancreatitis but had otherwise been well and living independently.
Initial diagnosis was thought to be sepsis secondary to a urinary tract infection with delirium, plus or minus carcinoid crisis.

She was commenced on short acting Octreotide to cover any carcinoid crisis and Tazocin for the urinary tract infection. This therapy resulted in slightly improved inflammatory markers but continued delirium. CT head ruled out brain metastasise as a cause of confusion. Other possible causes investigated included hypothyroidism and slight hyponatremia $(125 \mathrm{mmol} / \mathrm{L})$. She was treated with Thyroxine and the hyponatremia monitored for any further decline. Ammonia levels were also tested to rule out an encephalopathic cause of her confusion, these were reported normal.

Whilst admitted this patient became increasingly more confused and oedematous, probably secondary to hypoalbuminemia. She developed a peau d'orange rash. As this patient displayed signs of diarrhoea, confusion and rash a diagnosis of Pellagra was postulated and she was treated with Vitamin B Co-strong, Pabrinex and Multivitamins. She also had extensive input with dieticians who attempted to increase her protein intake to help with her hypoalbuminemia.

Unfortunately this patient did not do well on treatment and passed away comfortably with her family at her side.

\section{Discussion}

Carcinoid tumours affect 2.47-4.4/100,000 people with a mean age of diagnosis being 61.4years (1). Intestinal carcinoid tumours are most commonly found in the appendix, terminal ileum and rectum with common extra-intestinal tumours being found in lung, testis and ovary. They are classed as carcinoid by their cell origin, enterochromaffin cells and can be functioning or non-functioning. The tumours are slow growing and commonly remain asymptomatic until metastasise are present (2). Less than $10 \%$ of patients with a carcinoid tumour present with carcinoid syndrome (1), a combination of symptoms including hypotension, intermittent abdominal pain, diarrhoea, flushing, palpitations and wheeze, predicting a poorer outcome than those patients who remain asymptomatic (3). Diagnosis and disease progression is often monitored with hormone levels, such as Serotonin. Raised serotonin levels in twenty-four hour urine collection are $73 \%$ sensitive and $100 \%$ specific for carcinoid diagnosis. Levels are seen to decrease on treatment with somatostatin analogues (Octreotide), $50 \%$ reduction being indicative of biochemical response (4). Management of carcinoid tumours range from surgical resection of the tumour, chemotherapy, embolisation of liver metastasise and medical therapies such as Octreotide that can provide symptomatic relief (4). Prognosis of carcinoid tumours worsens as disease progresses (50.4\% 5 year survival rate) with poorer prognosis being seen in those with metastatic disease $(21.8 \% 5$ year survival rate (5). If the tumour can be resected surgically prognosis is greatly improved ( $83 \% 5$ year survival rate) $(6)$.

Carcinoid crisis is recognised as an acute onset of flushing, bronchospasm, tachycardia and fluctuating blood pressure and can occur during anaesthetic induction or during invasive procedures such as surgery, embolisation and radiofrequency ablation. This is thought to be secondary to the release of large amounts of serotonin and other vasoactive peptides from the tumour. This makes intervention extremely risky and should always be overseen by an experienced anaesthetist (7). Patients are often given prophylactic Octreotide prior to intervention to prevent a carcinoid crisis (5).

Pellagra is commonly associated with carcinoid syndrome and can sometimes be the presenting factor that leads to diagnosis. It is a severe deficiency in Niacin (Vitamin B-3) or Tryptophan, from which niacin can be synthesised. It is clinically characterised by photosensitive dermatitis, diarrhoea, dementia and death. Niacin is involved in oxidation-reduction reactions required for cellular function, being an essential component of nicotinamide coenzymes (8). The deficiency can be primary, due to reduced niacin levels in the diet or secondary due to reduced absorption of niacin in the gastrointestinal tract as a result of gastrointestinal disease. The primary form of this deficiency is more commonly seen in the developing world where the major food source is corn. Diagnosis is most commonly seen in adults and it affects both sexes equally (9). If pellagra is left untreated death is inevitable from multi organ failure. Management is with Niacin supplement therapy but if secondary pellagra is present and malnutrition evident then supplementation of B-complex vitamins and a high protein diet is essential. Death is unavoidable if treatment is not commenced (3).

\section{Learning Points}

- Rare Causes of confusion like Vitamin deficiency should be considered in all cases when no other obvious cause is identified.

- Pellagra should be considered in patients with Carcinoid syndrome.

- Pellagra is due to deficiency of Niacin and if left untreated can lead to death.

\section{References}

1. Barakat M.T, Meeran K, Bloom S.R. Neuroendocrine Tumours. Endocr Relat Cancer. 2004 Mar; (1) : 1-18

2. Kumar P, Clark M. Clinical Medicine. 6ed. 2005.

3. Kulke MH. Clinical presentation and management of carcinoid tumours. Hematol Oncol Clin North Am. 2007;21:433-455

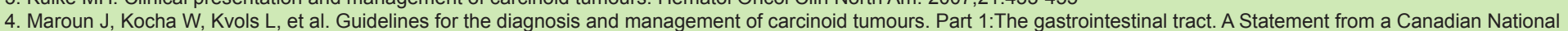
Carcinoid Expert Group. Current Oncology. 13(2);April 2006. (p67 - 76.)

5. Modlin, I. M. and Sandor, A. An analysis of 8305 cases of carcinoid tumors. Cancer, 1997. (79): 813-829.

6. Shebani K.O, Souba W.W, Finkelstein D.M, et al. Prognosis and Survival in Patients With Gastrointestinal Tract Carcinoid Tumours. Ann Surg. 1999 June $229(6): 815$.

7. Ramage JK, Davies AHG, Ardill J et al. Guidelines for the management of gastroenteropancreatic neuroendocrine (including carcinoid) tumours. Gut 2005;54(Suppl IV):

8. Pocock G, Richard C.D. Human Physiology. The Basis of Medicine. 3ed. 2006.

9. Heygyi V. Dermatological Manifestations of Pellagra. Updated July 2010. http://emedicine.medscape.com/article/1095845-overview [Accessed 16/4/2012]. 